4. Auswaschen in dest. Wasser.

5. Waschen in $0,1-m$. Citratpuffer von $p_{\mathrm{H}} 4,6$. Dauer: 1/2-1 Minute.

6. Auswaschen in dest. Wasser.

7. Danach in farblosem Schwefelammonium (10 Tropfen konzentriert auf $\left.100 \mathrm{ml} \mathrm{H}_{2} \mathrm{O}\right)$. Dauer: 1 bis 3 Minuten.

8. Überführen der Präparate in 1-proz. $\mathrm{OsO}_{4}$ $\left(p_{\mathrm{H}} 7,2\right)$. Dauer: 2 Stunden.

9. Danach übliche Entwässerung und Einbettung in Methacrylat.

Die nach der obrigen Vorschrift behandelten Gewebe zeigen im Ultradünnschnitt eine relativ gute Strukturerhaltung. Aus den Untersuchungen von Goмов ${ }^{2}$, Schulte und Eger ${ }^{1}$ ist bereits bekannt, daß die Phosphoamidase fast ausschließlich in den Zellkernen und den Grenzflächen lokalisiert ist. So ließ sich auch elektronenmikroskopisch mit dieser Methode in den Zellkernen der Endothelzellen (Abb. ${ }^{*}$ ) und in den Zellkernen der Glomerulischleifen das an dem Ort des Enzyms durch

\section{Einfluß von Temperatur und Salzgehalt auf Myomerenzahl und Körpergröße von Heringslarven}

\author{
Von Gotthilf Hempel
}

Biologische Anstalt Helgoland, Helgoland

und John H. S. Blaxter

Marine Laboratory, Aberdeen

(Z. Naturforschg. 16 b, 227-228 [1961] ; eingeg. am 19. November 1960)

Die auf Helgoland durchgeführten Aufzuchtversuche an Heringsbrut boten Gelegenheit zur Fortsetzung früherer Experimente über die Modifikabilität der Myomerenzahl des Herings. Im Hauptversuch ${ }^{1}$ waren die künstlich befruchteten Eier unterschiedlicher Temperatur, aber einheitlichen Licht- und Salzgehaltbedingungen ausgesetzt. In einer weiteren Versuchsserie wurden bei gleicher konstanter Temperatur unterschiedliche Salzgehalte $(5-50 \%)$ geboten. Als Elterntiere dienten Frühjahrslaicher aus der Elbmündung und der Kieler Bucht, auch wurden die Eier eines Kieler Herings mit dem durch Tiefgefrieren konservierten Sperma ${ }^{2}$ des an der Westküste Schottlands (Clyde) laichenden Herings befruchtet. Proben zu je 50 Larven wurden nach dem Schlüpfen und nach Resorption des Dotters fixiert. Die Zählung der Myomeren erfolgte in polarisiertem Licht ${ }^{3}$. In ihm sind die Myomeren des Schwanzes weit besser sichtbar als bei der früher geübten Zählung ${ }^{4}$ an lebenden Larven in normalem Licht. Die Befunde bestätigten statistisch gesichert die von HeMPEL ${ }^{4}$ und Blaxter ${ }^{3}$ beschriebene negative Relation zwischen der mittleren Myomerenzahl frisch geschlüpfter Herings-

1 J. H. S. Blaxter u. G. Hempel, Naturwissenschaften 48, 81 [1961].

2 J. H. S. Blaxter, Nature [London] 172, 1189 [1953]. die Behandlung gebildete Bleisulfid nachweisen (Abb. 2). Von gewissem Nachteil erweist sich die Partikelgröße des Reaktionsproduktes. Es kann darum keine Aussage über die Bindung des Enzyms an bestimmte Strukturen im Zellkern gemacht werden (Abb. 3). Übereinstimmend mit Eger und Schulte ${ }^{1}$ konnten auch wir feststellen, daß nicht alle Zellkerne im Gewebe gleichen Reaktionsanfall zeigen. Neben solchen Kernen, die sehr deutlich das Reaktionsprodukt aufweisen, finden sich ebensoviele ohne irgendeine Enzymreaktion. Vornehmlich im Muskelgewebe blieben die Kerne der Muskelzellen in der Reaktion negativ, während die Kerne der gefäßführenden Zellen dicht mit dem Reaktionsprodukt ausgefüllt waren. In einer späteren ausführlichen Veröffentlichung sollen die an den einzelnen Organen erhobenen Befunde und die unterschiedlichen Reaktionsnormen der verschiedenen Zelltypen besprochen werden.

* Abb. 1-3 s. Tafel S. 224 a u. b.

larven und der Erbrütungstemperatur (Abb. 1 a). Mit dem Salzgehalt ist die Myomerenzahl positiv korreliert $(A b b .1$ b). Hat man die großen Unterschiede in der mittleren Wirbelzahl der Elternpopulationen im Auge $(55,2$ bzw. 57,0$)$, so überrascht, daß die Larven des Küstenherings kaum weniger Myomeren aufweisen als die Clyde-Larven ${ }^{3}$ und daß die Kiel $\times$ Clyde-Kreuzung nur geringfügig (aber signifikant) höhere Myomeren-
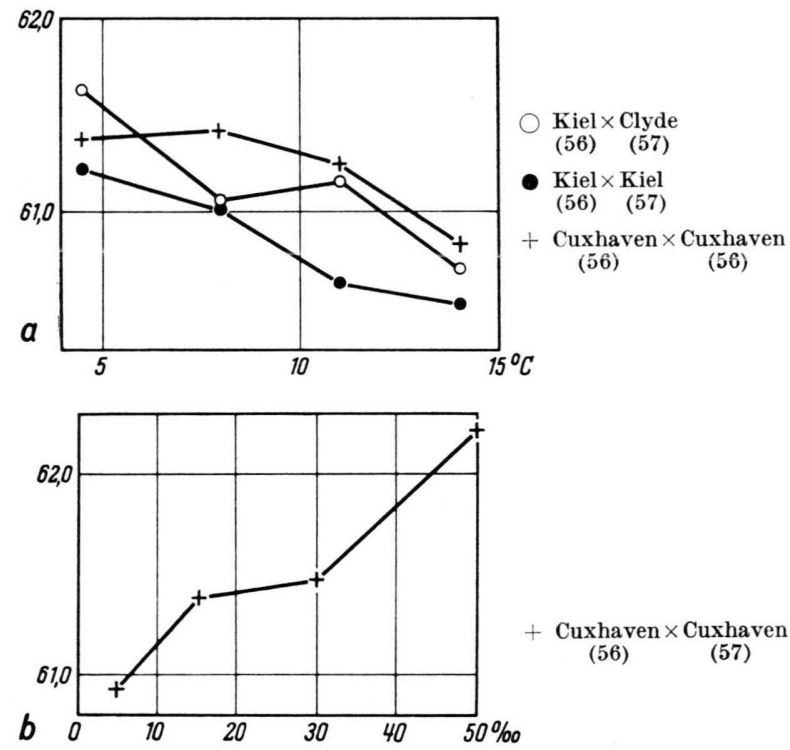

Abb. 1. Mittlere Myomerenzahlen frischgeschlüpfter Heringslarven in Abhängigkeit von der Temperatur (a) und vom Salzgehalt (b). Wirbelzahlen der Elterntiere in Klammern.

3 J. H. S. Blaxter, Marine Res. Scot. 1957, 1.

4 G. Hempel, Naturwissenschaften 40, 467 [1953]. 

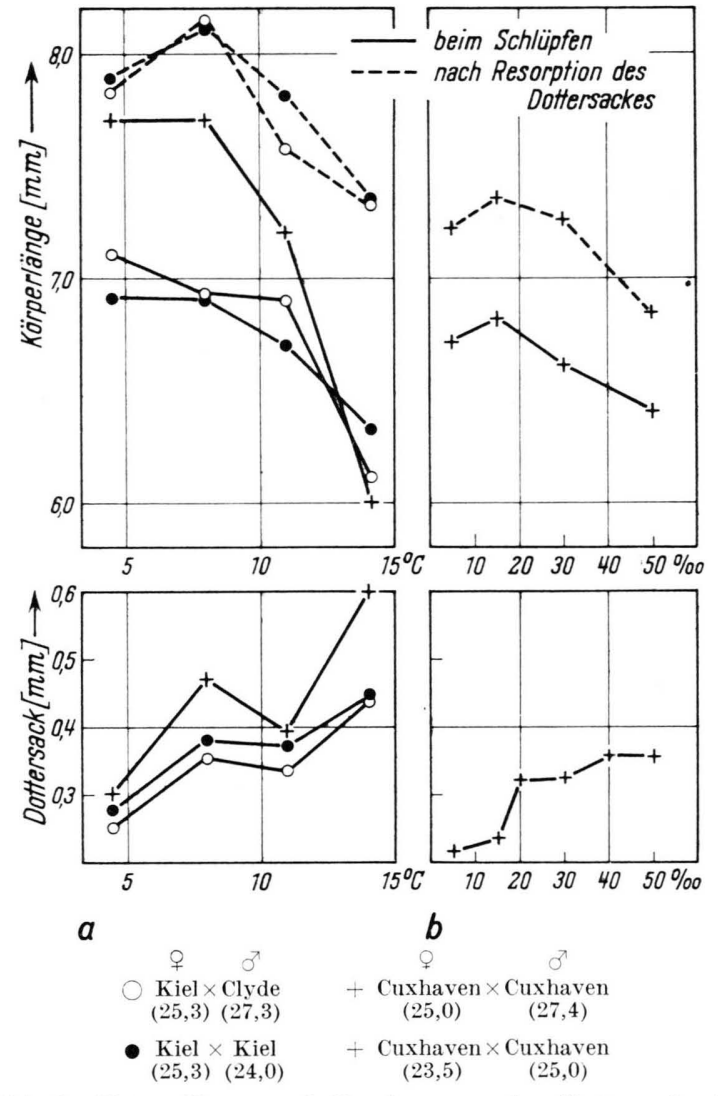

Abb. 2. Körperlänge und Durchmesser des Dottersacks in Abhängigkeit von Temperatur und Salzgehalt. Körperlänge der Eltern in Klammern.

zahlen bei gleichen Erbrütungsbedingungen erbrachte als die Kiel $\times$ Kiel-Larven. Allerdings hatten die für die Versuche verwendeten deutschen Küstenheringe zufällig überdurchschnittlich hohe Wirbelzahlen. Innerhalb der einzelnen Proben fand sich keine klare Beziehung zwischen der Körpergröße und der Myomerenzahl der Larven.

Nach der Resorption des Dotters ist es oft schwierig, die Myomeren in der Kopf- und Schwanzregion sicher zu erkennen. Das gilt besonders für die bei $5{ }^{\circ} \mathrm{C}$ erbrüteten Larven, die - wie in früheren Versuchen recht hinfällig waren. Wir gewannen den Eindruck, daß die mittleren Myomerenzahlen am Ende des Dottersackstadiums nicht mehr so deutlich mit der Temperatur korreliert sind. Vielmehr fanden wir bei mittleren Temperaturen niedrigere Werte als bei den Extremtemperaturen. Weitere Zählungen sind erforderlich, um diese Verhältnisse zu klären.

In Fortsetzung der Aufzuchtversuche ergab sich bei den in $8^{\circ}, 11^{\circ}$ und $14^{\circ} \mathrm{C}$ erbrüteten und später bei einheitlicher Temperatur gehaltenen Larven eine negative Beziehung zwischen Temperatur und Wirbelzahl $(P<0,05)$. Dieser Befund sollte aber an einem gröBeren Material erhärtet werden.

Körperlänge und Dotterreichtum der Larven sind von der Temperatur und dem Salzgehalt während der Erbrütung abhängig (Abb. 2). Am Ende des Dottersackstadiums waren die bei $8{ }^{\circ} \mathrm{C}$ und die bei $15^{\circ} / 00 \mathrm{~S}$ erbrüteten Larven am größten. Die Inkubationszeit wurde vom Salzgehalt nicht deutlich beeinflußt, wohl aber die Befruchtungs- und Schlüpfraten. Sie waren bei der Brut der Küstenheringe im Gegensatz zu derjenigen Her Nordsee-Bankheringe im Bereich mittlerer Salzgeh: $(15-20 \% 00)$ am günstigsten. $\mathrm{Ob}$ dies und die Unterschiede in der Körpergröße als Zeichen einer Anpassung an die Umweltbedingungen auf dem Laichplatz zu werten sind, ist noch ungeklärt.

Die Aufzuchtversuche werden ausführlicher in den Helgoländer Wissenschaftlichen Meeresuntersuchungen dargestellt, die Ergebnisse der Myomerenzählungen voraussichtlich im Journal du Conseil Internat. Explor. Mer. 